\title{
Long telomeres: A new prognostic factor for severity in triple-negative breast cancer patients
}

\author{
Oriol Calvete $^{1,2 *}$, Silvana Mouron ${ }^{3}$, Alicia Barroso ${ }^{1}$, Nora Soberon ${ }^{4}$, Maria A Blasco ${ }^{4}$, Miguel Quintela-Fandino ${ }^{3}$ and Javier Benitez ${ }^{1,2}$ \\ ${ }^{1}$ Human Genetics Group, CNIO-Spanish National Cancer Research Centre. Madrid, Spain \\ ${ }^{2}$ Network of Research on Rare Diseases (CIBERER). Madrid, Spain \\ ${ }^{3}$ Breast Cancer Clinical Research Unit, CNIO-Spanish National Cancer Research Centre, Madrid, Spain \\ ${ }^{4}$ Telomeres and Telomerase Group, Molecular Oncology Programme, CNIO-Spanish, National Cancer Research Centre, Madrid, Spain
}

\begin{abstract}
Abnormal telomere length is involved in several cancer types. However, it is not well understood how telomere length can be used as a prognostic and/or predictive factor in breast cancer or for the identification of its different molecular subtypes.

Here, we evaluate the telomere length of a series of 105 treatment-naive HER2-negative patients (80 luminal and 25 triple negative) to assess the correlation of telomere size with the clinical course of breast cancer subtypes.

Our results revealed the existence of a subgroup of 17 patients with long telomeres; most of their tumours were of the triple-negative subtype, had the highest histological grade and a high Ki67 proliferation index, suggesting an association between long telomeres and cancer aggressiveness. Specifically, long telomeres and reduced telomere shortening correlated with triple-negative patients. In addition, patients with long telomeres had a significantly earlier age of onset, the highest Ki67 proliferation index, and the worst response to treatment compared to all bad responder patients. Long-telomere tumours also showed increased immunohistochemical staining of PS6 such as POT1 mutation carriers. Taken together, our findings seem to define a new subgroup of more aggressive triple-negative cases (around 40\%) associated with long telomeres and reduced telomere shortening. More studies and follow-up are necessary to validate these preliminary results.
\end{abstract}

\section{Introduction}

Telomeres play an important role in various cancer types, where gradual telomere shortening associated with mitotic division leads to cell senescence and apoptosis in early steps of transformation [1]. Abnormal telomere length (TL) may have prognostic significance of malignancy and cancer risk. There is growing evidence from cancer susceptibility and population studies that points to a correlation between genetic variants that shorten TL and an increased risk of cancer [2]. By contrast, reduced telomere shortening induced by mutations in the POT1 gene, encoding a member of the shelterin complex involved in telomere maintenance, lead to long telomeres and tumour progression in several cancer types such as chronic lymphocytic leukaemia, colorectal cancer, melanoma, glioma and angiosarcoma [3]. In breast cancer, TL has been frequently evaluated but previous studies did not establish robust prognostic and/or predictive correlations between TL and any of the three main molecular subtypes [4]. Previously, short but not long telomeres were suggested to be associated with more aggressive breast cancer subtypes [5]. TL does not seem to be associated with increased breast cancer risk either [6,7]. Lack of significant evidence can be explained by the recent observation that chemotherapy affects TL [8]. Thus, treatment-naive patients should be considered in the studies that aim to correlate TL with disease susceptibility or clinical course, since chemotherapy-modified TL can bias the true biological associations.

\section{Materials and methods}

Patients: TL of a HER2-negative breast cancer series was previously measured [9]. The initial series was composed of peripheral blood of
130 treatment-naïve HER2-negative breast cancer patients. Of this series, we re-evaluated the TL of 105 patients with complete clinical information; 80 patients were luminal (oestrogen receptor -ER- and/or progesterone receptor-PR- positive and HER2-negative) and 25 patients were triple negative (Table S1). TL in peripheral blood mononuclear cells prior to chemotherapy treatment was measured by qPCR.

Informed consent was obtained for all patients included in this study. All study procedures were conducted in accordance with the Declaration of Helsinki and Good Clinical Practice standards. Ethics Committee from Hospital Univesitario Ramon y Cajal (Madrid, Spain), approved this study (ID of the approval: CEIC REF. 04.082011).

Targeted next generation sequencing (tNGS): Thirteen triple negative patients from the studied series (10 Ltel and 3 Ntel patients) were sequenced by tNGS using the Onco4 Gene Panel from Sistemas Genómicos (Supplementary Materials and Methods). Twenty-five ng of index case genomic DNA was tagmented and amplified. Quality and quantity of purified libraries were assessed with an Agilent 2100 Bioanalyzer instrument. Libraries were sequenced on a MiSeq platform

*Correspondence to: Oriol Calvete, Spanish National Cancer Research Centre, (CNIO). Melchor Fernández Almagro, 3. 28029, Madrid, Spain, E-mail: ocalvete@cnio.es

Key words: long telomeres, breast cancer risk, triple-negative subtype, telomere shortening rate, POT1

Received: November 04, 2020; Accepted: November 19, 2020; Published: November 22, 2020 
from Illumina. Reads were aligned against the human reference genome version GRCh38/hg38 using BWA aligner. Low-quality reads and PCR duplicates were removed from the BAM-formatted file at this point. Variant calling was performed using a combination of VarScan and GATK, along with in-house scripts to combine and filter variants. Only variants in coding regions with a minor allele frequency $(\mathrm{MAF})>0.01$ that were putatively deleterious as measured by Sift, Polyphen 2 and Condel algorithms were considered. Identified variants were annotated using the Ensembl database. The candidate variants were confirmed by Sanger sequencing.

Immunohistochemistry studies: Formalin-fixed paraffinembedded (FFPE) tissue samples were obtained from patients' biopsies and were cut into 5 - $\mu \mathrm{m}$-thick sections and stained with Hematoxylin and Eosin (H\&E) for light microscopy examination. FFPE blocks were cut into 5-1 m-thick sections for immunohistochemistry (IHC) studies. Proliferation index in tumours was tested by counting the percentage of the cells of the tumours stained with Ki67 antibody from DAKO (Ref:IR626). PI3K/Akt pathway was tested with anti-PS6 ribosomal protein (Ser235/236) from Cell Signaling Technology (Danvers, MA; Ref. 2211) following the manufacturers' instructions. Both the absence of staining and excess nonspecific staining were considered as negative staining

\section{Results}

Telomere length in HER2-negative breast cancer patients: Recently, the TL of a HER2-negative breast cancer series was measured to evaluate its relationship with chemotherapy treatment toxicity(9). Median TL was not associated with toxicity but the percentage of critically short telomeres $(<3 \mathrm{~Kb})$ correlated with greater toxic episodes associated with paclitaxel (fatigue, myalgia and neuropathy). On the other hand, TL prior to chemotherapy treatment measurements revealed a number of outliers within the series with abnormally long telomeres; thus, we re-evaluated the influence of TL on several clinical features related to aggressive behaviour to better understand the role of telomeres in HER2-negative breast cancer.

The initial series was composed of peripheral blood of 130 treatment-naïve HER2-negative breast cancer patients that were scheduled for neoadjuvant chemotherapy with paclitaxel. Of this series, we studied 105 patients with complete clinical information; 80 patients were luminal (oestrogen receptor -ER- and/or progesterone receptor-PR-positive and HER2-negative) and 25 patients were triple negative (Table S1). TL was measured in peripheral blood mononuclear cells prior to chemotherapy treatment (Observed TL) and normalized for age (Expected TL) using TL in a control population [8] (Table 1).
Differences between Observed/Expected TL were included in a linear regression model, which showed a lower slope in cases $(R 2=0.204$; $P=0.033)$ compared to the normal population ( $\mathrm{R} 2=0.574 ; P=0.001$ ). Some of the patients presented an increased TL compared with the control population (Figure 1A). We selected these patients with increased TL by identifying those with a positive TL ratio (Observed compared to Expected measurement) and a reduced percentage (less than $10 \%$ ) of short telomeres (less than $3 \mathrm{~Kb}$ ), which correlates with a reduced telomere shortening rate in cells [1]. Seventeen of the 105 patients were classified in the long telomeres (Ltel) group, while the remaining 88 patients were considered to have normal-sized telomeres (Ntel) (Figure 1B). Both TL and percentage short telomeres were statistically significantly different $(\mathrm{p}<0.001)$ between Ltel and Ntel groups (Table 1).

\section{Comparison between clinical features of long (Ltel) and normal (Ntel) telomeres patient groups}

The comparison between Ltel and Ntel groups (Table 1) showed that 10 of the 25 triple negative (TN) patients were included in the Ltel group, which represents $58.82 \%$ of patients of the Ltel group, while only 7 of the 17 Ltel patients were luminal $(41.18 \%)$. By contrast, the Ntel group contained only $15 \mathrm{TN}$ patients (17.05\%) and 73 patients $(82.95 \%)$ belonging to the luminal group. The proportion of TN subtype was significantly higher in the group with long telomeres than in the Ntel group ( $\mathrm{p}=0.004)$ (Table 1 ) and suggests the existence of a TN subgroup having long telomeres (Figure 1C). The histologic grade (grades 1, 2 or 3) was compared within groups. Grade 3 patients were the most represented in the Ltel group (58.82\%), while only $22.73 \%$ of patients from the Ntel group were classified with this grade (Figure 1D). The percentage of patients for each grade was significantly different between Ltel and Ntel groups $(\mathrm{p}<0.001)$ and suggested a positive correlation between TL and histologic grade (Table 1). Similarly, the median ratio of TL (Observed:Expected) increased with the tumour grade (Figure 1E). Regarding the Ki67 proliferation index, which is the strongest negative prognostic factor for luminal tumors [10], an average of $48.75 \%$ and $28.77 \%$ of the cells of the tumours stained positive in the Ltel and the Ntel groups, respectively (Figure $1 \mathrm{~F}$ ), which means higher proliferation rates in patients with long telomeres $(\mathrm{p}=0.016$; Table 1$)$. On the other hand, the average age of diagnosis was lower in patients included in the Ltel group (45.84 years old) compared to the Ntel group (50.03 years old) $(\mathrm{p}=0.060)$, although this difference was borderline significant.

Finally, different asymptotic significances (bilateral) were calculated using contingency tables to evaluate the independence of clinical comparisons within the HER2-negative series (Table 1). Long telomeres and a reduced number (less than 10\%) of critically short telomeres

Table 1. Average telomere length and clinical information of the Ntel and Ltel groups

\begin{tabular}{|c|c|c|c|c|c|c|c|c|c|c|c|c|}
\hline & & \multicolumn{4}{|c|}{ Telomere length $(\mathrm{Kb})$} & \multicolumn{2}{|c|}{ Receptors } & \multicolumn{3}{|c|}{ Grade } & \multirow[b]{2}{*}{ Ki67 (\%) } & \multirow[b]{2}{*}{$\begin{array}{l}\text { Age dx. } \\
\text { (years) }\end{array}$} \\
\hline & $\mathbf{N}$ & Expected & Observed & Difference & $\begin{array}{c}\%<3 k b \\
\text { (Tshort) }\end{array}$ & TN- (\%) & $\mathrm{ER} / \mathrm{PR}+(\%)$ & $1(\%)$ & $2(\%)$ & $3(\%)$ & & \\
\hline Total & 105 & 9.3 & 9.84 & 0.54 & 17.38 & $25(23.81)$ & $80(76.19)$ & 12 (11.42) & $63(60.0)$ & $30(28.57)$ & 31.85 & 49.35 \\
\hline Ntel & 88 & 9.26 & 9.2 & -0.06 & 19.14 & $15(17.05)$ & $63(82.95)$ & $11(12.50)$ & $57(64.77)$ & $20(22.73)$ & 28.77 & 50.03 \\
\hline Ltel & 17 & 9.54 & 13.15 & 3.61 & 8.23 & $10(58.82)$ & $7(41.18)$ & $1(5.88)$ & $6(35.29)$ & $10(58.82)$ & 48.75 & 45.84 \\
\hline \multirow{2}{*}{\multicolumn{3}{|c|}{$\begin{array}{l}\text { pValue } \\
\text { pValue }^{o o}\end{array}$}} & $<0.001 * * *$ & $<0.001 * * *$ & $<0.001 * * *$ & \multicolumn{2}{|c|}{$0.004^{* *}$} & \multicolumn{3}{|c|}{$<0.001^{* * *}$} & $0.016^{*}$ & \multirow{3}{*}{0.060 (NS } \\
\hline & & & & $<0.001 * * *$ & & & & $<0.0$ & $* * *$ & & & \\
\hline \multicolumn{3}{|l|}{ pValue $\mathrm{e}^{\mathrm{ooo}}$} & \multicolumn{9}{|c|}{$<0.01^{*}$} & \\
\hline
\end{tabular}

TN: triple negative; ER: Oestrogen receptor; PR: Progesterone receptor; */**: Significant; NS: no significant

o: Significance between Ntel /Ltel groups

${ }^{\circ}$ : Asymptotic significance (bilateral) for independence test within telomere length and clinics parameters

${ }^{\circ o 0}$ : Asymptotic significance (bilateral) for independence test between telomere length and clinics parameters 
A

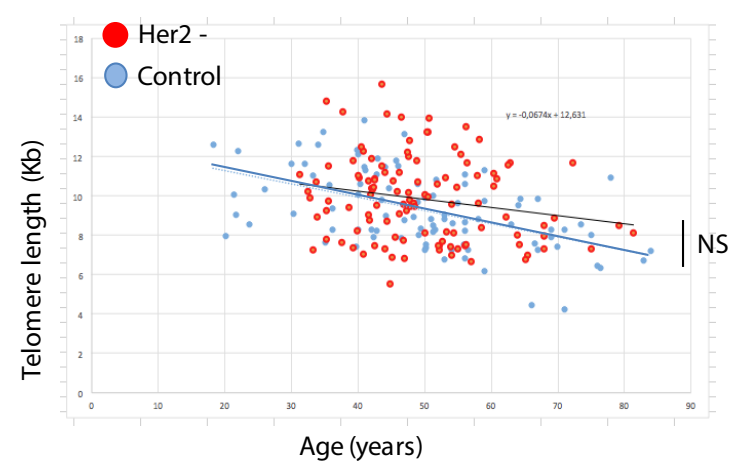

B

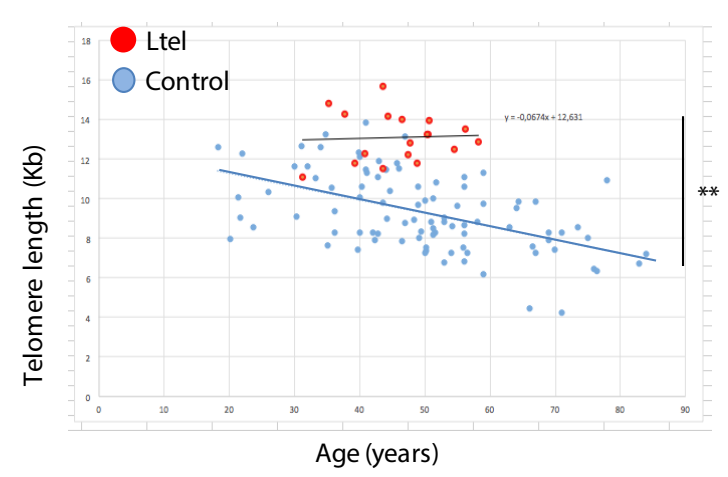

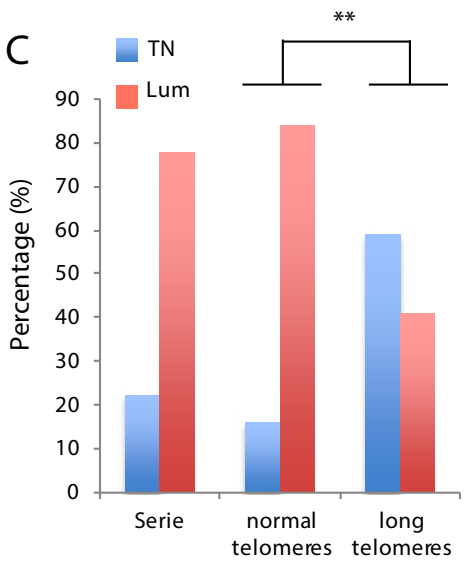

D

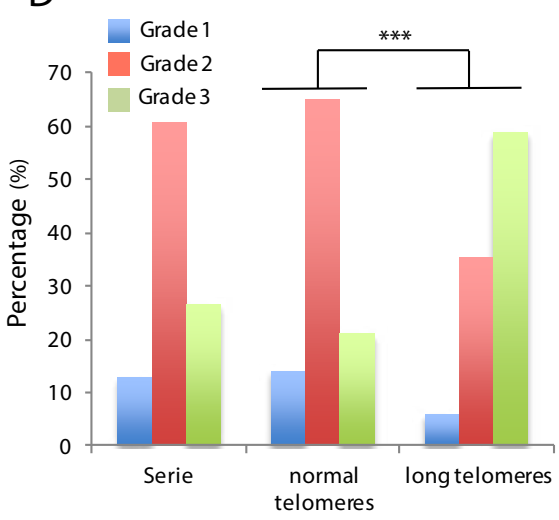

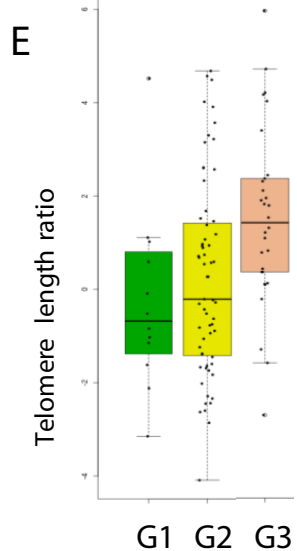

$\mathrm{F}$

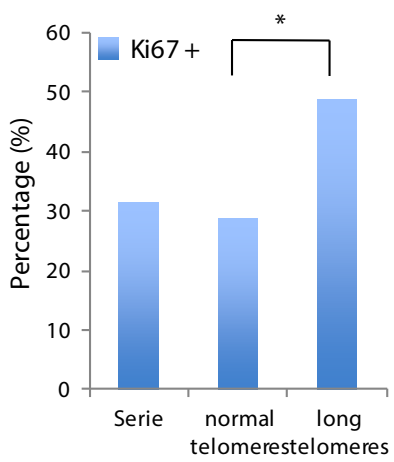

Figure 1. Telomere length (TL) and clinical evaluation of the HER2-negative breast cancer series. (A) TL (Kb) as a function of age (years) in the HER2-negative series (red dots) and the control population (blue dots). Regression lines are shown for the HER2-negative series (red) and the control population (blue). Differences between the HER2-negative series and the control population were not significant. (B) $\mathrm{TL}(\mathrm{Kb})$ as a function of age (years) in the HER2-negative patients with long telomeres and reduced telomere shortening (Ltel group; red dots) and the control population (blue dots). Regression lines are shown for the Ltel patients (red) and the control population (blue). (C) Percentage of triple negative (TN) and luminal (Lum) patients in the complete HER2-negative series, and Ntel and Ltel groups (** $\mathrm{p}=0.004)$. (D) Percentage of grade of malignancy (G1, G2 or G3) of tumours in the complete HER2-negative series, and the Ntel and Ltel groups (*** p<0.001). (E) Median of the telomere length ratio (Observed:Expected) of the patients from the complete HER2-negative series per tumour grade (G1, G2 or G3). (F) Average percentage of the tumour cells positively stained with Ki67 in patients of the complete HER2-negative series, and Ntel and Ltel groups (* p=0.016)

(less than $<3 \mathrm{~Kb})$ were positively associated $(\mathrm{p}<0.001)$, suggesting that long telomeres were present due to the lack of normal shortening in somatic cells. Similarly, tumour subtype classification, histologic grade and Ki67 were also observed to be positively correlated $(\mathrm{p}<0.001)$. Finally, TL measurements (long telomeres and a reduced number of short telomeres) and clinical features (molecular subtype, grade and proliferation) were positively correlated $(\mathrm{p}<0.01)$ in the series, suggesting an association between aggressiveness and long telomeres. Age of diagnosis did no correlate with TL or clinical features (Table 1).

Telomere differences within triple negative and luminal subtypes: No differences in telomere shortening rate (regression lines slope) were observed between triple negative (TN) and luminal (Lum) patients (Figure 2A). Likewise, within-group comparisons showed that Ltel patients had normal telomere shortening rate compared to Ntel patients in the Lum group (Figure $2 \mathrm{~B}$ ). However, telomere shortening rate was reduced in Ltel patients compared to the Ntel patients within the TN group (Figure 2C). Within the TN group, Ltel patients had an earlier age of onset (47.74 years) than Ntel patients (52.00 years). Regarding the Ki67 proliferation index, $67.30 \%$ and $49.27 \%$ of the cells of the tumours were positively stained in the Ltel and the Ntel groups, respectively. These results suggest a positive correlation between early diagnosis and highly proliferative tumours in patients with long telomeres within the TN subtype (Table S2).
The cohort of patients was treated with single-agent paclitaxel in the neoadjuvant setting, and pathologic complete response was determined according to the Symmans and Pusztai method [11]. Patients obtaining a score of 0 or 1 ( $>90 \%$ cure rate in the long term) were classified as good responders, whereas those with higher scores $(>30 \%$ relapse rate in the long term) were classified as bad responders. Around $80 \%$ of patients of both subgroups (TN_Ltel and TN_Ntel) were bad responders to the used treatment. However, bad responders with long telomeres (Bad_ Ltel) had an earlier age of onset (Figure 2D) and their tumours had higher Ki67 proliferation rates (Figure 2E) than the bad responders with normal telomeres (Bad_Ntel).

Because between $10-15 \%$ of TN patients present mutations in the $B R C A 1$ or $B R C A 2$ genes [12], the 10 cases from the LTel group were studied by targeted Next Generation Sequencing (tNGS) with the Onco4 Gene Panel from Sistemas Genómicos. Only one case (10\%) with a BRCA1 mutation (BRCA1p.Pro968LeufsTer33; rs398122670) was found, suggesting that this subgroup of TN with long telomeres is not associated with BRCA1/2 mutations.

Molecular validation of long telomere pathogenicity: Long telomeres and reduced telomere shortening have been described in cancer patients with mutations in the POT1 gene, a telomere maintenance gene [3]. Recently, we described that tumours from POT1 

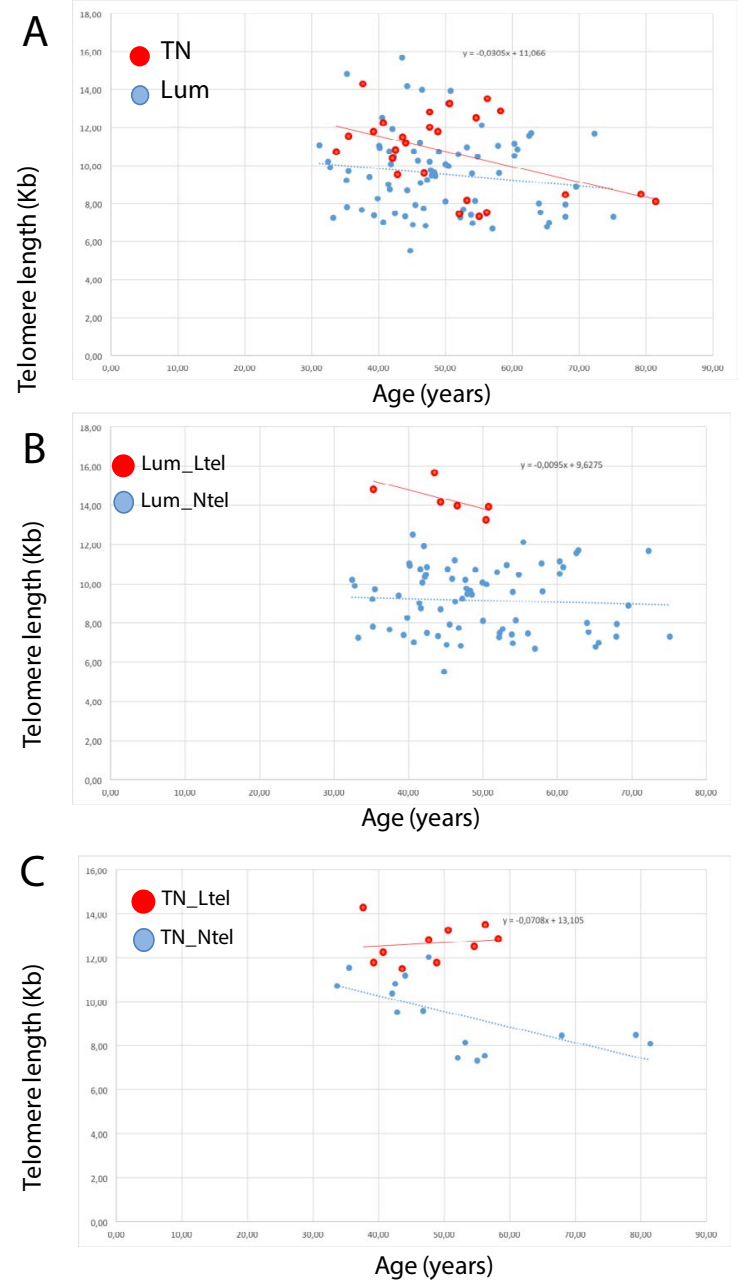

Figure 2. Comparison of telomere length (TL) and clinical features of Ntel and Ltel patients. (A) TL (Kb) as a function of age (years) for triple negative (TN; red dots) and luminal (blue dots) patients. No significant differences were observed for regression lines for the TN (red) and Lum (blue) subtypes. (B) TL (Kb) as a function of age (years) for luminal patients with long telomeres (Ltel; red dots) and normal telomeres (Ntel; blue dots). Regression lines are shown for the Ltel (red) and Ntel (blue) patients. (C) TL (Kb) as a function of age (years) for triple negative (TN) patients with long telomeres (Ltel; red dots) and normal telomeres (Ntel; blue dots). Regression lines are shown for the Ltel (red) and Ntel (blue) patients. (D) Age at diagnosis of the patients from the complete HER2-negative series as a function of treatment response (NS: not significant; $* * p<0.01 ; * * * p<0.001$ ). (E) Percentage of cells in tumour stained with Ki67 for the patients of the complete HER2-negative series as a function of treatment response (NS: not significant; ${ }^{* *} \mathrm{p}<0.01 ; * * * \mathrm{p}<0.001$ ). (F) Representative anti-PS6 antibody staining of tumour tissue of a HER2-positive breast cancer patient (control), an Ltel patient (HER2-negative, grade 2, 50\% Ki67) and an Ntel patient (HER2-negative, grade 2, 70\% Ki67)

mutation carriers exhibited increased immunohistochemical staining of PS6, a downstream molecule of the PI3K/Akt pathway [13]. Thus, in order to correlate long telomeres in our series with alterations in telomere maintenance function, we evaluated our HER2-negative series of patients with anti-PS6 staining. We selected 4 Ltel and 4 Ntel formalin-fixed paraffin-embedded (FFPE) grade 2 tumour tissues with high Ki67 staining from our series. Four other FFPE tumour tissues from HER2-positive breast cancer patients with normal telomeres were included in the study as controls. Increased anti-PS6 staining was observed in Ltel patients compared to the Ntel group and HER2-positive breast cancer controls (Figure 2F). Since both Ltel and Ntel HER2negative tumours were grade 2 with high Ki67 staining, the observed increased anti-pS6 staining difference correlates with telomere length differences. Increased anti-PS6 staining in Ltel patients is in agreement with our previous observation in patients with long telomeres driven by mutations in the POT1 gene [13]. In order to test the possible role of the POT1 gene in this TN subgroup with long telomeres, POT1 mutations were evaluated from the tNGS data. No mutations in the POT1 gene were found.

\section{Discussion}

In summary, we describe a new group of TN patients with long telomeres and reduced telomere shortening rate that significantly correlated with more aggressive characteristics (higher grade and proliferation rate, and earlier age of onset). This subgroup represents around $40 \%$ of TN cases. Previously, short but not long telomeres were suggested to be associated with more aggressive breast cancer subtypes [5]. In addition, our results suggest that reduced telomere shortening may correlate with worse response to treatment.

In addition, increased anti-PS6 staining was observed in tumours from patients of the studied series with long telomeres, which is in agreement with previous observations in other tumour types with altered POT1 function and long telomeres [3,13]. However, no mutations in the POT1 gene were found in our series of patients, suggesting that other genes involved in telomere maintenance might explain the telomere length alterations and breast cancer risk. 
Finally, only one mutation was found in the BRCA1 gene in the TN Ltel group, which seems to indicate that there is no association between long telomeres and BRCA genes. Thus, larger studies in TN breast cancer patients with long telomeres are necessary in order to decipher the aetiology, clinical and genetic features and treatment response associated with this subgroup with a more aggressive pathology.

\section{Financial support}

OC received funding from the H2020 BRIDGES project (Number: 634935). JB's lab is partially funded by grant PI16/00440 from the Instituto de Salud Carlos III (ISCIII), co-funded by the European Regional Development Fund (ERDF). MQF is a recipient of the following grants: PI16/00354 funded by the ISCIII and co-funded by the ERDF, and B2017/BMD3733 (Immunothercan-CM) - Call for Coordinated Research Groups from Madrid Region - Madrid Regional Government - ERDF funds. Research in the Blasco lab is funded by the Spanish Ministry of Economy and Competitiveness Projects (SAF201345111-R and SAF2015-72455-EXP), the Comunidad de Madrid Project (S2017/BMD-3770), the World Cancer Research (WCR) Project (161177) and the Fundación Botín (Spain).

\section{Competing interests}

The authors declare that they have no competing interests.

\section{References}

1. Zhu X, Han W, Xue W, Zou Y, Xie C, et al. (2016) The association between telomere length and cancer risk in population studies. Sci Rep 3: 636-651. [Crossref]

2. Haycock PC, Burgess S, Nounu A, Zheng J, Okoli GN, et al. (2017) Association between telomere length and risk of cancer and non-neoplastic diseases a mendelian randomization study. JAMA Oncol 3: 636-651. [Crossref]
3. Calvete O, Garcia-Pavia P, Domínguez F, Bougeard G, Kunze K, et al. (2017) The wide spectrum of POT1 gene variants correlates with multiple cancer types. Eur J Hum Genet 4: 1-4. [Crossref]

4. Ennour-Idrissi K, Maunsell E, Diorio C (2017) Telomere length and breast cancer prognosis: A systematic review. Cancer Epidemiol Biomarkers Prev 4: 3-10. [Crossref]

5. Heaphy CM, Subhawong AP, Gross AL, Konishi Y, Kouprina N, et al. (2011) Shorter telomeres in luminal B, HER-2 and triple-negative breast cancer subtypes. Mod Pathol 24: $194-200$.

6. Luu HN, Long J, Wen W, Zheng Y, Cai Q, et al. (2016) Association between genetic risk score for telomere length and risk of breast cancer. Cancer Causes Control 27: 1219-1228.

7. Pavanello S, Varesco L, Gismondi V, Bruzzi P, Bolognesi C, et al. (2018) Leucocytes telomere length and breast cancer risk/ susceptibility: A case-control study. PLoS One 13: e0197522 [Crossref]

8. Benitez-Buelga C, Sanchez-Barroso L, Gallardo M, Apellániz-Ruiz M, Inglada- Pérez L, et al. (2015) Impact of chemotherapy on telomere length in sporadic and familial breast cancer patients. Breast Cancer Res Treat 49: 385-394.

9. Quintela-Fandino M, Soberon N, Lluch A, Manso L, Calvo I, et al. (2017) Critically short telomeres and toxicity of chemotherapy in early breast cancer. Oncotarget 8: 21472-21482. [Crossref]

10. Cheang MCU, Chia SK, Voduc D, Gao D, Leung S, et al. (2009) Ki67 index, HER2 status, and prognosis of patients with luminal B breast cancer. J Natl Cancer Inst 101: 736-750. [Crossref]

11. Symmans WF, Peintinger F, Hatzis C, Rajan R, Kuerer H, et al. (2007) Measurement of residual breast cancer burden to predict survival after neoadjuvant chemotherapy. $J$ Clin Oncol 25: 4414-4422. [Crossref]

12. Easton DF, Pharoah PDP, Antoniou AC, Tischkowitz M, Tavtigian SV, et al. (2015) Gene-panel sequencing and the prediction of breast-cancer risk. N Engl J Med 372: 2243-2257.

13. Calvete O, Garcia-Pavia P, Domínguez F, Mosteiro L, Pérez-Cabornero L, et al. (2019) POT1 and damage response malfunction trigger acquisition of somatic activating mutations in the VEGF pathway in cardiac angiosarcomas. J Am Heart Assoc 8: e012875. [Crossref]

Copyright: (C2020 Calvete O. This is an open-access article distributed under the terms of the Creative Commons Attribution License, which permits unrestricted use, distribution, and reproduction in any medium, provided the original author and source are credited. 